\title{
MicroRNA-663 facilitates the growth, migration and invasion of ovarian cancer cell by inhibiting TUSC2
}

Hui Hui Xie ${ }^{1 \dagger}$, Wen Ting Huan ${ }^{1 \dagger}$, Jiang Qiong Han², Wei Ru Ren ${ }^{3}$ and Li Hua Yang ${ }^{4^{*}}$

\begin{abstract}
Background: MicroRNAs (miRNAs) have emerged as the critical modulators of the tumorigenesis and tumor progression.

Methods: The levels of miR-663 in ovarian cancer cell lines and clinical tissues were detected using qRT-PCR assays. The Transwell invasion and wound healing assay were conducted to assess the roles of miR-663 in the migration and invasion of ovarian cancer cell in vitro. Rescue assays were carried out to confirm the contribution of tumor suppressor candidate 2 (TUSC2) in the aggressiveness of cancer cell which was regulated by miR-663.

Results: The levels of miR-663 were up-regulated in ovarian cancer tissues in comparison with the corresponding normal tissues. Up-regulation of miR-663 increased the proliferation, colony formation, migration and invasion of ovarian cancer SKOV3 cell. Additional, over-expression of miR-663 increased the tumor growth of SKOV3 in xenograft model. Bioinformatics analysis and luciferase reporter assay identified that miR-663 decreased the level of TUSC2 via binding to the 3'-UTR of TUSC2 gene. Finally, the expression of TUSC2 was inversely associated with the level of miR663 in ovarian carcinoma tissue and over-expression of TUSC2 inhibited the migration and invasion abilities of SKOV3 that was promoted by miR-663.
\end{abstract}

Conclusion: Altogether, these results indicate that miR-663 acts as a potential tumor-promoting miRNA through targeting TUSC2 in ovarian cancer.

Keywords: MiR-663, Ovarian cancer, TUSC2, Growth, Migration, Invasion

\section{Background}

Ovarian carcinoma is one of the commonly gynecological malignancies with unoptimistic clinical outcomes [1, 2]. Although the remarkably improvement in the treatment options for ovarian cancer in the last few years, the 5-year survival rate of this disease is not optimistic, mainly due to advanced stage at diagnosis and the recurrence and metastasis [3, 4]. Ovarian cancer often metastasizes throughout the peritoneal cavity, to the omentum, and even to the parenchyma of the liver or lung. Many

\footnotetext{
*Correspondence: yanglihua0628@foxmail.com; yanglihua0628@163. com

${ }^{\dagger}$ Hui Hui Xie and Wen Ting Huan contributed equally to this work

${ }^{4}$ Department of Gynaecology, the 2nd Affiliated Hospital of Kunming

Medical University, Kunming, Yunnan, China

Full list of author information is available at the end of the article
}

investigations have indicated that the metastasis of ovarian cancer cell could be divided into several steps: (I) cell detachment, survival, and resistance of anoikis; (II) evasion of immunological surveillance; (III) epithelialmesenchymal transition; (IV) spheroid formation; (V) ascites formation; and (VI) peritoneal implantation [5, 6]. Because $>70 \%$ of ovarian cancer cases are diagnosed at a late stage, when cancer cells are actively metastasizing, understanding ovarian cancer pathogenesis and the mechanism of its metastasis is crucial for the management of this deadly, highly metastatic disease [7]. Hence, elaborating the novel-innovative mechanism of ovarian cancer metastasis are crucial to exploitation new therapeutic strategy [8].

MicroRNAs (miRNAs), which belong to a kind of conserved noncoding RNAs, regulate the expressions of 
target proteins in posttranscriptional dependent manner through binding to $3^{\prime}$-untranslated region ( $3^{\prime}$-UTR) of genes [9]. The roles of alternated miRNAs in diverse biological processes, including tumor cell growth, cellular apoptosis and chemotherapy resistance have been exactly demonstrated [10]. MiRNAs function as tumor suppressor or oncogenic miRNA in the tumorigenesis and cancer progression based on the functions of their target genes [11]. Previous studies have shown the role of miR-663 in many important pathological processes, including autoimmune diseases, infection, and inflammatory reaction $[12,13]$. However, its function in tumor progression is contradictory. Although it acts as a cancer promoter in breast cancer and nasopharyngeal carcinoma, miR-663 may also be shown as a potential tumor suppressor in glioblastoma and gastric cancer [14, 15]. However, to our knowledge, no functional evidence of miR663 in ovarian carcinoma has been documented.

Tumor suppressor candidate 2 (TUSC2), which is also known as FUS1 in chromosome 3p21.3 region, has been identified to function as a anti-oncogene [16]. Allele losses and genetic alterations of TUSC2 are found in multitudinous cancer types, including lung carcinoma and breast cancer [17]. For example, TUSC2 serves as a tumor-suppressor via up-regulating the level of miR-197 in glioblastoma [18]. Currently, TUSC2 is down-regulated in nasopharyngeal carcinoma (NPC) genes and relates to cellular apoptosis in the chromosome deletion regions. Nevertheless, the expression of TUSC2 gene in ovarian cancer remains not well investigated.

In this study, we demonstrated that miR-663 was upregulated in ovarian cancer. Furthermore, over-expression of miR-663 promoted the growth, migration and invasion of ovarian cancer cell. In addition, TUSC2 was identified as the target gene of miR-663 and its expression was negatively regulated by miR-663. Finally, we found that miR-663 promoted the aggressiveness of ovarian carcinoma cellvia targeting TUSC2.

\section{Methods}

\section{Cell lines and tissue samples}

Ovarian carcinoma cells (SKOV3, HO-8910, OVCAR, A2780) and the ovarian surface epithelial cell line, HOSEpiC, were purchased from Jennio Biotech Co., Ltd (GuangZhou, Guangdong, China). Cells were cultured with 1640 or DMEM (Invitrogen, Carlsbad, CA, USA) containing $10 \% \mathrm{FBS}$ and $1 \%$ penicillin/streptomycin. Twenty-three pairs of ovarian cancer tissues and adjacent normal tissues were obtained from Gynaecology Ward of Maternal and Child Health Hospital (Zaozhuang, Shandong Province, China) and Xuzhou Central Hospital. Prior patients' consent and ethics approval were obtained from the Gynaecology Ward of Maternal and Child
Health Hospital and Xuzhou Central Hospital. All tissues were immediately frozen in liquid nitrogen for future tests.

\section{Transfection}

SKOV3 cell line was selected for investigating the role of miR-663 in ovarian carcinoma owing to the level of miR-663 was highest in SKOV3. Cells at confluency of 40-50\% were transfected with miRNAs $(5 \mu \mathrm{g} /$ well for 6 well culture plates) using Lipofectamine 2000 reagent (Invitrogen; Carlsbad, Calif, USA). The miR-663 mimic and a nonspecific miR control (miR-NC) were purchased from GenePharma (Shanghai, China). The expression construct of TUSC2 was produced by subcloning PCR-amplied full-length human TUSC2 cDNA into the pCDNA3.1 plasmid (Invitrogen, Carlsbad CA, USA). For cotransfection, SKOV3 cells were transiently transfected with plasmids ( $2 \mu \mathrm{g} /$ well for 6 well culture plates) containing the TUSC2 and miR-663 mimic $(5 \mu \mathrm{g} /$ well for 6 well culture plates) using Lipofectamine 2000 reagent (Invitrogen, Carlsbad, Calif, USA) according to the manufacturer's recommendations.

\section{Quantitative real-time PCR}

RNA was extracted using TRIzol reagent (Invitrogen CA, USA). RNA $(1 \mu \mathrm{g})$ was reversely transcribed into cDNA using the PrimeScript RT reagent kit (TakaraBio, Tokyo, Japan). qRT-PCR was conducted using SYBR Premix Ex Taq ${ }^{\mathrm{TM}}$ kit (TakaraBio) on the Applied Biosystems 7900 Sequence Detection system (Applied Biosystems). For detecting miR-663, the Mir-VanaTM MiRNA Isolation Kit (Ambion, USA) was used to isolate total RNA from cell lines and patient samples following the manufacturer's instructions. MiR-663 was detected using Platinum Taq DNA Polymerase (Invitrogen) on the Applied Biosystems 7900 Sequence Detection system (Applied Biosystems). The primers were as follows: TUSC2 (forward primer: 5'-GGAGACAATCGTCAC CAAGAAC-3'; reserve primer: 5'-TCACACCTCATA GAGGATCACAG-3'), U6 (forward primer: 5'-AAA GCAAATCATCGGACGACC-3'; reverse primer: 5'-GTACAACACATTGTTTCCTCGGA-3'), TGF $\beta 1$ (forward primer: 5'-GTAGCTCTGATGAGTGCA ATGAC-3'; reverse primer: 5'-CAGATATGGCAACTC CCAGTG-3'); eEF1A2 (forward primer: 5'-GAAGAC CCACATCAACATCGT-3'; reverse primer: 5'-CTC CGCATTTGTAGATGAGGTG-3'); HRAS (forward primer: $\quad 5^{\prime}$-TGCTTCAGTTTGAACTACCCTG-3'; reverse primer: $5^{\prime}$-GCCCAGTGCTGATAGCCAG-3'); PIK3CD (forward primer: $5^{\prime}$-AAGGAGGAGAATCAG AGCGTT-3'; reverse primer: 5'-GAAGAGCGGCTC ATACTGGG-3'); CXCR4 (forward primer: 5'-ACTACA CCGAGGAAATGGGCT-3'; reverse primer: $5^{\prime}$-CCC 
ACAATGCCAGTTAAGAAGA-3'); GAPDH (forward primer: 5'-TGTGGGCATCAATGGATTTGG3'; reverse primer: 5'-ACACCATGTATTCCGGGT CAAT-3'). U6 forward: CTCGCTTCGGCAGCACA, U6 reverse: AACGCTTCACGAATTTGCGT. U6 and GAPDH were the internal controls. The reaction condition was $95{ }^{\circ} \mathrm{C}$ for $5 \mathrm{~min}$, followed by 40 cycles of denaturation at $95{ }^{\circ} \mathrm{C}$ for $15 \mathrm{~s}$ and annealing/elongation step at $60{ }^{\circ} \mathrm{C}$ for $30 \mathrm{~s}$. The comparative cycle threshold $(\mathrm{Ct})$ method was selected to detect the level by calculating the $2^{(-\Delta \Delta C t)}$.

\section{Cells proliferation analysis}

SKOV3 cell growth in vitro was detected using CCK-8 (Beyotime, Nanjing, Jiangsu, China) method. $2 \times 10^{3}$ SKVO3 cells were cultured in 96-well plate for continued $24 \mathrm{~h}, 48 \mathrm{~h}, 72 \mathrm{~h}$ or $96 \mathrm{~h}$, respectively. After each time node, each well was added CCK-8 solution $(10 \mu \mathrm{L})$ and after $1 \mathrm{~h}$, the OD was detected at $450 \mathrm{~nm}$.

\section{Clone formation analysis}

500 cells/well miR-663 or miR-NC transfected ovarian carcinoma SKOV3 cells were plated into 6-well plate, and cells were cultured for 2 weeks. Cell colonies were stained using $1 \%$ crystal violet and the number of cell colonies was counted.

\section{Cell migration assay}

$1 \times 10^{5} /$ well transfected cells were grown in six-well plates and formed confluence. The cell confluence was swiped using a $100 \mu \mathrm{L}$ tip to generate a gap. The SKOV3 cells wound were observed at 0 and $24 \mathrm{~h}$ using a ZEISS invert microscope (CarlZeiss, Germany) [19].

\section{Cell invasion analysis}

Cell invasiveness was detected using the Transwell invasion assay. $1 \times 10^{5}$ SKOV3 cells were plated into Matrigel coated upper chamber of Transwell. Medium containing 20\% FBS was plated into lower chamber of Transwell. After $24 \mathrm{~h}$, invaded SKOV3 cells were fixed with $4 \%$ methyl aldehyde and stained using $1 \%$ crystal violet. The number of invaded cell was counted in five randomly fields [20].

\section{Luciferase reporter analysis}

3'-UTR-TUSC2 gene containing the binding site of miR663 was PCR amplified and then sub-cloned into the psiCHECK2 (Promega, Madison, WI, USA). Mutant type $3^{\prime}$-UTR-TUSC2 gene was established using the Agilent Technologies QuikChange XL Site Directed Mutagenesis Kit and sub-cloned into the psiCHECK2. In luciferase reporter test, SKOV3 cells were co-transfected with miR-663 along with WT-UTR-TUSC2-3' or
MT-3'-UTR-TUSC2. The luciferase activity was measured in the luciferase reporter assay system (Promega).

\section{Immunoblotting}

Cells were lysed using RIPA (Beyotime, Nanjing, Jiangsu, China) and total protein was quantitated using the BCA kit (Thermo, Rockford, USA). Equal amounts of cell lysates $(25 \mu \mathrm{g})$ were loaded on $8 \%$ SDS-PAGE and transferred onto PVDF membranes.. The membranes were blocked with $5 \%$ nonfat dry milk in Tris-buffered saline with Tween 20 (TBST) for $1 \mathrm{~h}$ at $37{ }^{\circ} \mathrm{C}$. The membrane was incubated with antibodies against TUSC2 (1:1000, Santa Cruz Biotechnology, Dallas, Texas, USA) and internal control GAPDH (1:1000, Bioworld Technology, Nanjing, Jiangsu, China), followed by incubating with the HRP-linked secondary antibody $(1: 10,000$, Bioworld Technology, Nanjing, Jiangsu, China). The peroxidase activity was detected by ECL chemilumininescence detection kit (Pierce) and signal intensity of the protein bands were measured using the ChemiDoc XRS system (Bio-Rad, Hercules, CA, USA).

\section{Tumor xenograft model}

Animal experiment was approved by the Animal Research Committee of Xuzhou Central Hospital. Tumor xenograft model was established using BALB/c nude mice by subcutaneously injecting $2 \times 10^{6}$ miR-663 or miR-NC transfected SKOV3 cells. Tumor volume was monitored once every 3 days by assess the tumor width and length. The formula of tumor volume is $\left(\right.$ length $\times$ width $\left.^{2}\right) / 2$. After 25 days, tumor mass were exfoliated and weighed.

\section{Statistical analysis}

GraphPad Prism was used for statistical analysis. The data were presented as mean $\pm \mathrm{SD}$. Differences in the results of two groups were evaluated using either twotailed Student's $t$ test or one-way ANOVA followed by post hoc Dunnett's test. The differences with $P<0.05$ were considered statistically significant. The connection between TUSC 2 and miR-663 was tested by Spearman's correlation analysis.

\section{Results}

\section{MiR-663 is up-regulated in ovarian cancer}

To investigate the dysregulation of miRNAs in cervical cancer, the GEO dataset, GSE83693 that containing primary ovarian cancer tissue and normal ovarian tissue was selected to explore the expression patterns of miRNAs. As shown in Fig. 1a, the heatmap that was generated using differential levels of miRNAs indicated that miR-663 was significantly up-regulated in ovarian cancer 


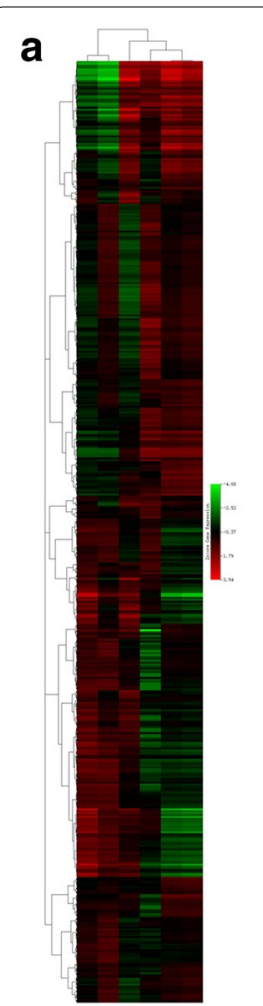

\section{b}

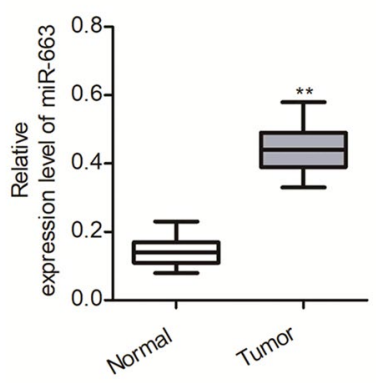

C

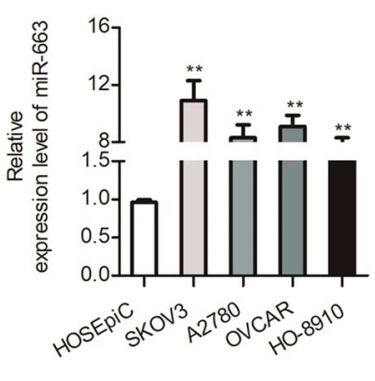

Fig. 1 The level of miR-663 is over-expressed in ovarian carcinoma. a Microarray analysis of miRNA expression in ovarian cancer tissues from normal control tissues. $\mathbf{b}$ The level of miR-663 in 23 paired ovarian cancer samples and matched normal tissues was assessed by qRT-PCR. ${ }^{* *} P<0.01$, compared to normal. c qRT-PCR assayed of miR-663 in ovarian carcinoma cells (OVCAR, SKOV3, A2780, and HO-8910) and HOSEpiC. $N=3$, ${ }^{* *} P<0.01$, compared with HOSEpiC cells

tissues. We then determined the levels of miR-663 in 23 pairs of ovarian cancer and corresponding non-cancer tissues using qRT-PCR method. As shown in Fig. 1b, miR-663 was significantly up-regulated in ovarian cancer tissues when compared with the matched normal tissues. Consistently, the level of miR-663 was higher in a panel of ovarian carcinoma cell lines (A2780, SKOV3, HO-8910 and OVCAR) than that in HOSEpiC (Fig. 1c). All these findings indicate that miR-663 is significantly up-regulated in both ovarian cancer samples and cell lines.

\section{Up-regulation of miR-663 promotes the proliferation of ovarian cancer cell}

To future explore the precise functions of miR-663 in ovarian carcinoma cell, SKOV3 cells were selected and transfected with miR-NC or miR-663 (Fig. 2a). CCK-8 experiment suggested that over-expression of miR-663 increased SKOV3 cell proliferation (Fig. 2b). Moreover, up-expression of miR-663 remarkably increased the colony formation of SKOV3 cell in vitro (Fig. 2c). To vitrify these observations, the miR-NC or miR-663 transfected SKOV3 cells were inoculated subcutaneously into nude mice. We found that the tumor growth was increased in mice that was inoculated with miR-663 transfected cells than that in the miR-NC group (Fig. 2d). Consistently, the Ki67 staining in tumor tissue that was formed by miR663 transfected SKOV3 cell was remarkably increased than that in the tumor tissue that derived from miR-NC transfected SKOV3 cell (Fig. 2e). All results uncover that over-regulation of miR-663 promotes ovarian cancer growth in vitro and in vivo.

\section{MiR-663 promotes ovarian cancer cell migration and invasion}

Given the correlation of cancer cell migration and distant metastasis in ovarian cancer, we further detected the biology effects of miR-663 on the migration and invasion of ovarian cancer cell. As shown in Fig. 3a, the wound healing assays suggested that miR-663 over-expression significantly increased the wound closure of SKOV3 cell in vitro. Consistently, over-expression of miR-663 promoted the invasion of SKOV3 cell compared to control cell (Fig. 3b).

\section{TUSC 2 is negative regulated bymiR- 663 in SKOV 3 cell}

Then, the targets of miR-663 were predicted using online analysis tools, including TargetScan (http://www.targe tscan.org), miRTarBase (http://mirtarbase.mbc.nctu. edu.tw/php/index.php) and miRDB (http://www.mirdb .org/). Total six common target genes were obtained from three bioinformatics analysis tools (Additional file 1: Figure S1A). In order to identify the direct gene of miR-663 the mRNA levels of these genes in miR-663 or miR-NC transfected SKOV3 cell were detected using qRT-PCR assay. As shown in Additional file 1: Figure S1B, the mRNA level of TUSC2 was significantly inhibited by miR-663 in SKOV3 cell. The complementary sequences of miR-663 were discovered in 3'-UTR of TUSC2 mRNA (Fig. 4a). Then, we conducted the luciferase analysis using miR-663 over-expressing SKOV3 cells that were transfected with wild type (WT) or mutant type (MUT) of TUSC2-3'-UTR. As shown in Fig. 4b, the luciferase activity of cells that were cotransfected with miR-663 and WT-TUSC2-3'-UTR was reduced whereas the luciferase activity was not affected in cells that were cotransfected with MUT TUSC2-3'-UTR and miR-663. Consistently, up-regulation of miR-663 inhibited the mRNA and protein level of TUSC2 in ovarian carcinoma SKOV3 cell (Fig. 4c, d). Evidence to date indicates that TUSC2, also known as FUS1, behaves as a tumor suppressor in several types of cancers, including lung cancer, breast cancer and thyroid cancer [21, 22]. Previous investigations have indicated that ectopic expression of TUSC2 inhibited 


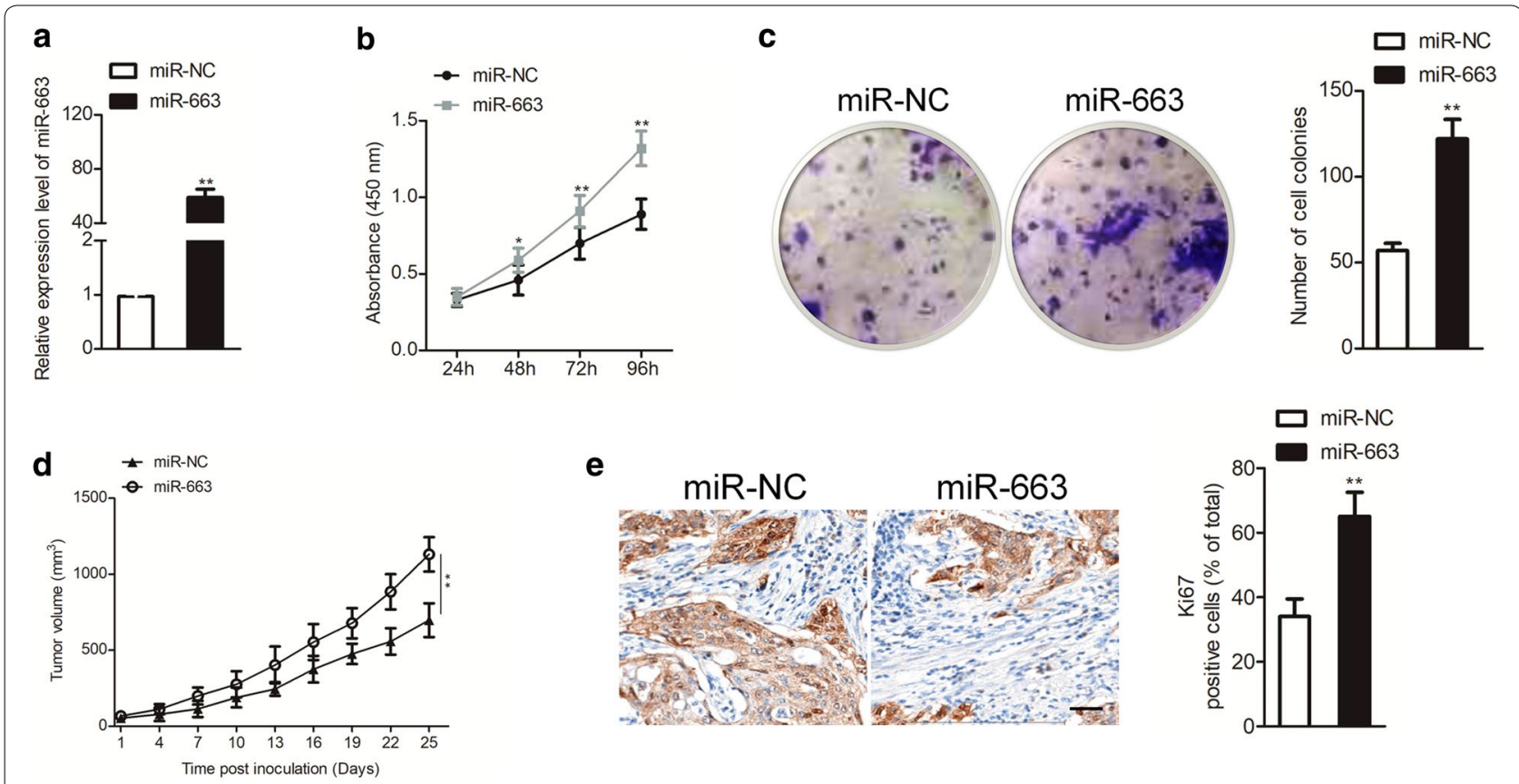

Fig. 2 MiR-663 facilitates ovarian carcinoma SKOV3 cells growth in vitro and in vivo. a SKOV3 cells were transfected with either miR-663 or miR-NC, and the level of miR-663 was measured using qRT-PCR method. $\mathbf{b}$ The proliferation of miR-663 or miR-NC transfected SKOV3 cells was detected by CCK-8 assay. c Cells colonies in either miR-663 or miR-NC transfected SKOV3 cells. $\mathbf{d}$ Nude mice were subcutaneously inoculated with miR-NC or miR-663 transfected SKOV3 cells and xenograft tumor volumes were measured. e Representative image of Ki67 immunohistochemical staining in indicated xenograft tumors. $\mathrm{N}=3,{ }^{* *} P<0.01$ in comparison to miR-NC

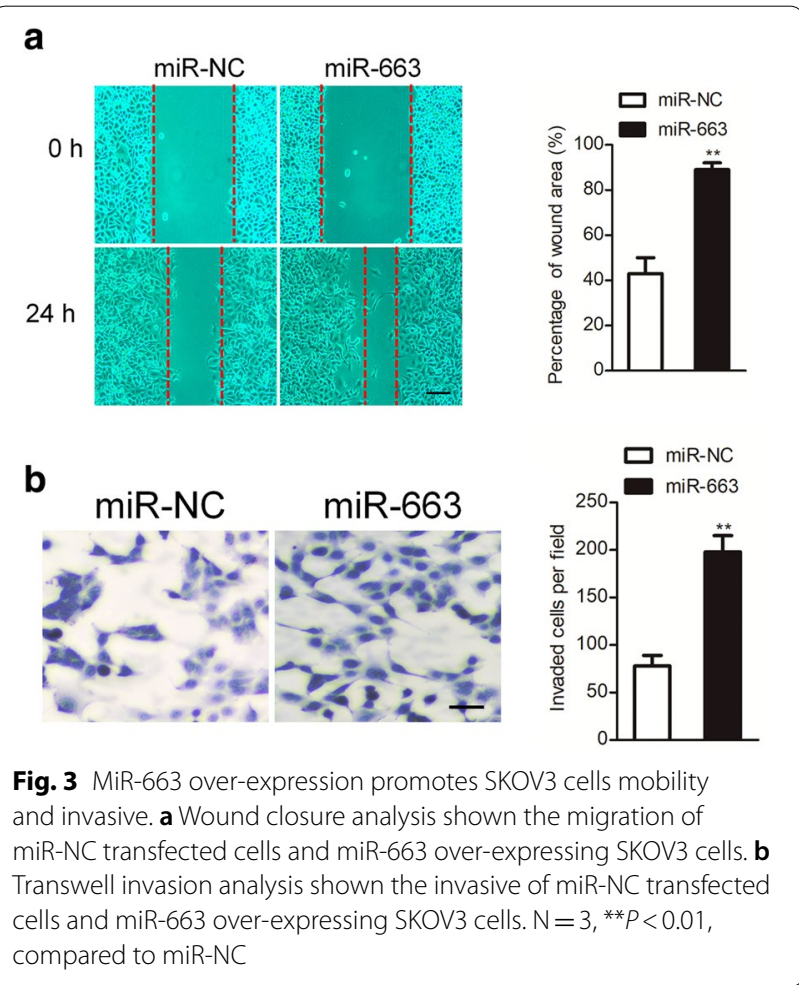

cell proliferation, survival, migration, and invasion, and increased tumor cell death [23]. Science the expression of TUSC 2 was negatively regulated by miR-663, we speculated that miR-663 promoted the growth, migration and invasion of SKOV3 via inhibiting the level of TUSC2.

\section{The effect of miR-663 on SKOV3 cell was inhibited by over-expression of TUSC 2}

To future investigate the actions of TUSC2 in miR663 mediated SKOV3 growth, migration and invasion, SKOV3 ovarian carcinoma cells were co-transfected with pCDNA3.1-TUSC2 and miR-663 mimics. The level of TUSC2 in SKOV3 cells was restored in SKOV3 cells that were cotransfected with TUSC2 over-expressing plasmid and miR-663 mimics (Fig. 5a, b). As expected, overexpression of TUSC2 significantly suppressed the SKOV3 cell growth, colony formation, migration and invasion that were induced by miR-663 (Fig. 5c-f). These findings reveal that miR-663 promotes ovarian cancer SKOV3 cell growth and metastasis by inhibiting TUSC2.

\section{TUSC2 is negatively correlated with miR-663 in ovarian cancer tissue}

Finally, we explored the level of TUSC2 in 23 pairs of ovarian carcinoma and matched normal tissues by qRTPCR assay. As shown in Fig. 6a, the levels of TUSC2 were 


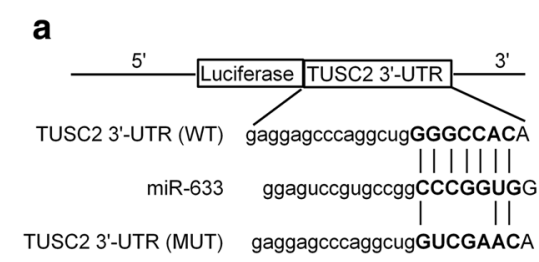

b

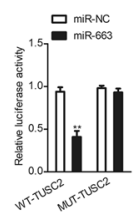

C

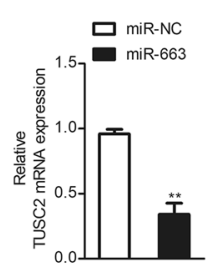

d

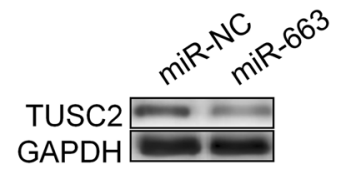

Fig. 4 TUSC2 is the target of miR-663 in SKOV3 cell. a Sequence alignment of wild-type and mutant type of miR-663-binding sites in 3'-UTR of TUSC2. $\mathbf{b}$ Luciferase activity was reduced in SKOV3 that co-transfected miR-663 along with WTTUSC2-3'-UTR. c The mRNA level of TUSC2 in miR-663 over-expressing SKOV3 was detected by qRT-PCR method. $\mathbf{d}$ Immunoblotting analysis of TUSC2 expression in miR-663 over-expressing SKOV3 cells. $N=3,{ }^{* *} P<0.01$, compared to miR-NC

lower in the ovarian cancer tissues in comparison with the adjacent normal tissues. Immunohistochemical staining analysis future proved that TUSC2 was down-regulated in ovarian cancer tissue compared to the matched normal tissue (Fig. 6b). Furthermore, the Spearman's correlation analysis showed that TUSC2 was negatively associated with miR-663 level in ovarian cancer (Fig. 6c). Altogether, these data demonstrate that TUSC2 is downexpressed and negatively associated with miR-663 in ovarian cancer tissue.

\section{Discussion}

MicroRNAs play vital roles in tumorigenesis and the progression of diverse cancers [24, 25]. MicroRNAs can regulate the expression of tumor related genes, and act as a tumor suppressor gene or an oncogene, playing an important role in the diagnosis and treatment of cancer [26]. In the current study, we explored the precise roles of miR-663 in ovarian cancer cell growth, migration and invasion. We found that miR-663 was up-regulated in ovarian carcinoma tissues and cell lines when compared to normal specimens and ovarian surface epithelial cell, respectively. Furthermore, over-expression of miR-663 promoted the growth, colony formation, migration and invasion of ovarian carcinoma SKOV3 cell in vitro. Consistently, the up-regulation of miR-663 increased the tumor growth of SKOV3 cell in vivo. Furthermore, we demonstrated that miR-663 facilitated ovarian cancer cell growth and progression by suppressing TUSC2.

Recently, there's increasing study and focus on the roles of miR-663 in the metastasis, chemotherapy-resistance and tumor-associated angiogenesis of various cancers. For example, miR-663 plays vital roles in nasopharyngeal carcinoma, pancreatic cancer and acute myeloid leukemia (AML) [27]. MiR-663 promotes nasopharyngeal carcinoma cells growth by means of directly targeting cyclin-dependent kinase inhibitor 2A (CDKN2A) and sustains non-small cell lung cancer (NSCLC) via restraining mitochondrial outer membrane permeabilization (MOMP) through p53 up-regulated modulator of apoptosis/Bcl-2 binding component 3 (PUMA/BBC3) and B-cell translocation gene 2 (BTG2). Nevertheless, the expression pattern and roles of miR-663 in ovarian carcinoma is not well investigated. In this study, we analyzed the levels of miR-663 in ovarian carcinoma tissues and demonstrated that up-expression of miR-663 facilitated the growth, migration and invasion of ovarian carcinoma SKOV3 cell. Bioinformatics analysis identified TUSC2 as the directly target of miR-663 and miR-663 over-expression inhibited the level of TUSC2 in ovarian cancer cell. The cancer suppressive functions of TUSC2 have been previously identified in other cancers. TUSC2 sensitizes non-small cell lung cancer cells to AKT inhibitor, MK2206 in LKB1 dependent manner [28]. Over-expression of TUSC2 inhibits the growth, mobility and invasion of glioblastoma cells [18]. In addition, TUSC2 is found to be down-regulated in nasopharyngeal carcinoma and negatively regulates proliferation and cell cycle progression [27]. On account of these observations, we speculated that miR-663 might participate into the malignant phenotype of ovarian carcinoma cells. Firstly, our data suggested that miR-663 was indeed up-regulated in ovarian carcinoma tissues when compared with control normal samples. Moreover, we demonstrated that overexpression of miR-663 remarkably facilitated the growth, mobility and aggressiveness of ovarian carcinoma cells. All these results were consistent with the previous study, in which miR-663b promotes the proliferation, migration and invasion of nasopharyngeal carcinoma cell through targeting TUSC2 [27]. Previous studies demonstrate that miRNAs negatively regulate protein expression through binding to $3^{\prime}$-UTR of target genes. In our data, luciferase reporter analysis suggested that miR-663 directly bind to $3^{\prime}$-UTR of TUSC2. What's more, restoration of TUSC2 neutralized the promotion of growth and migration in miR-663 transfected ovarian cancer cell. Since miR-663 plays such an important regulatory role in ovarian cancer, 


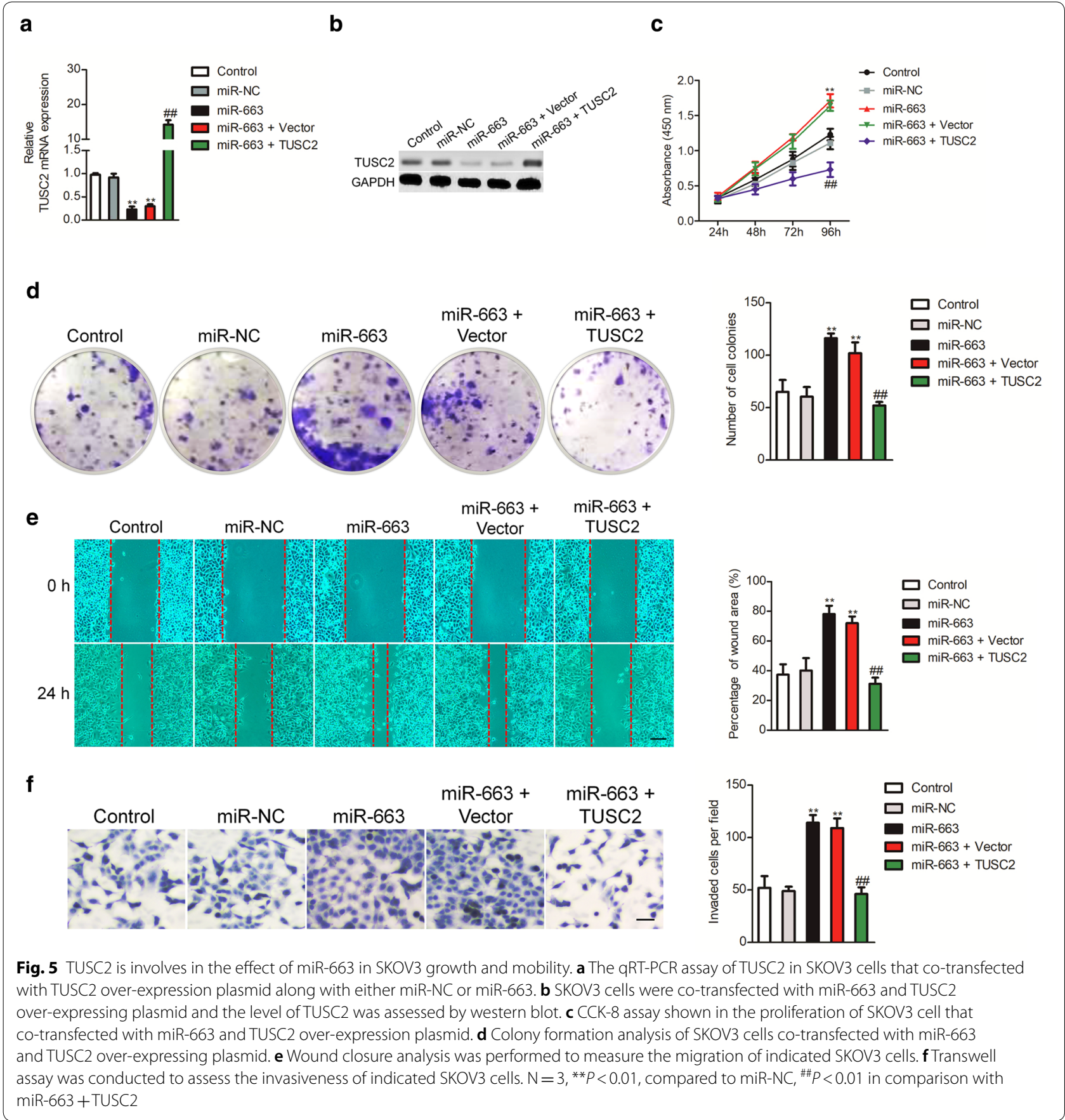

if we can develop a drug targeting miR-663 or interfering the interaction between miR-663 and downstream target gene, it may be able to provide a new therapeutic approach for the treatment of ovarian cancer. Of course, these assumptions need more experimental data to validate and more supports from clinical data. Meanwhile, the primary limitation of this work is that we only analyzed fewer ovarian cancer clinical tissues and cell lines. Hence, more precise investigations are needed for profoundly explore the latent therapeutic role of miR-663 in ovarian carcinoma. 


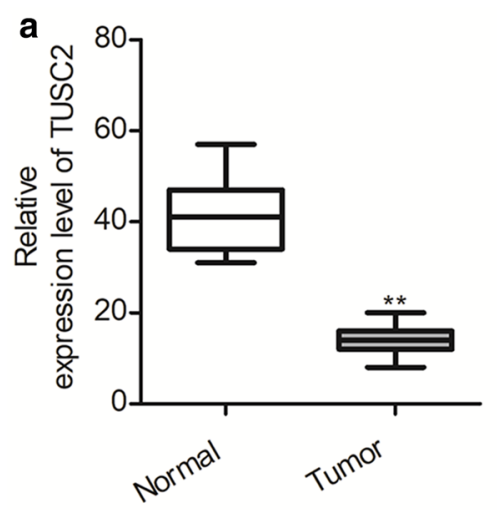

b

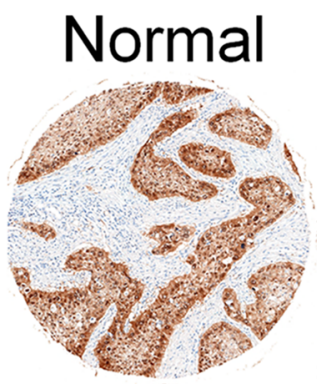

\section{Tumor}

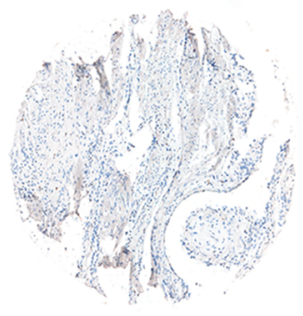

C
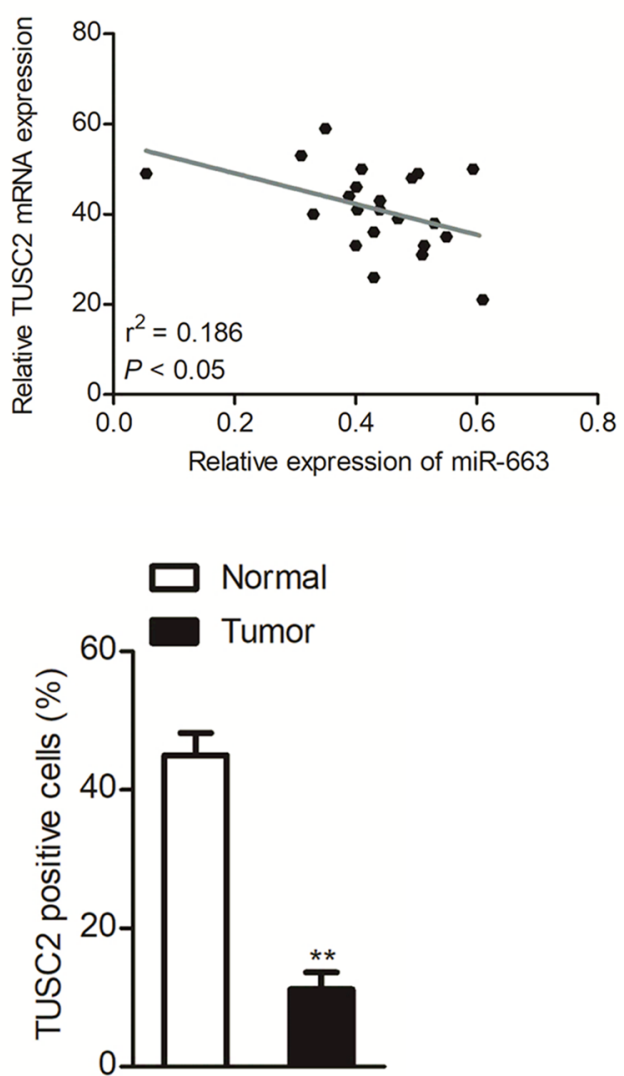

Fig. 6 TUSC2 is negatively associated with miR-663 in ovarian carcinoma. a The level of TUSC2 in 23 paired ovarian carcinoma and adjacent normal samples was detected by qRT-PCR. $\mathbf{b}$ Immunohistochemical staining of TUSC2 in ovarian carcinoma tissue samples and corresponding normal. $N=3$. ${ }^{*} P<0.01$, compared to normal. c Spearman's correlation analysis of the relationship of TUSC2 and miR-663 in ovarian carcinoma ( $n=23$ )

\section{Conclusion}

In conclusion, miR-663 promoted ovarian cancer cell growth and progression by targeting TUSC2. Furthermore, these results provided insight into the potential therapeutic value of miR-663 in reducing ovarian cancer cell growth and aggressiveness.

\section{Additional file}

Additional file 1: Figure S1. TUSC2 is the target of miR-663 in SKOV3 cell. A. Venn graph represented the number of candidate common target genes determined by three bioinformatics analysis. B. SKOV3 cell was transfected with miR-NC or miR-663 and the levels of potential target genes were measured by qRT-PCR assay. ${ }^{* *} P<0.01$ in comparison to control.

\section{Abbreviations}

TUSC2: tumor suppressor candidate 2; 3'-UTR: 3'-untranslated region; qRT-PCR: quantitative real-time PCR; GAPDH: glyceraldehyde-3-phosphate dehydrogenase; miR-NC: miR-negative control; DMEM: Dulbecco's modified Eagle medium; WT: wild type; MUT: mutant type; NSCLC: non-small cell lung cancer;
MOMP: mitochondrial outer membrane permeabilization; PUMA/BBC3: p53 up-regulated modulator of apoptosis/Bcl-2 binding component 3; BTG2: B cell translocation gene 2 .

\section{Authors' contributions}

HHX, WTH and WRR deigned and performed the whole study. JQH and LHY analyzed the data and wrote the paper. All authors read and approved the final manuscript.

\section{Author details}

${ }^{1}$ Obstetrics and Gynecology, Xuzhou Central Hospital, Xuzhou, Jiangsu, China.

${ }^{2}$ Department of Integrated Traditional Chinese and Western Medicine, The Third Affiliated Hospital of Kunming Medical University (The Tumor Hospital of Yunnan Province), Kunming, Yunnan, China. ${ }^{3}$ Gynaecology Ward of Maternal and Child Health Hospital, Zaozhuang, Shandong, China. ${ }^{4}$ Department of Gynaecology, the 2nd Affiliated Hospital of Kunming Medical University, Kunming, Yunnan, China.

\section{Acknowledgements}

Not applicable.

\section{Competing interests}

The authors declare that they have no competing interests. 


\section{Availability of data and materials}

All data generated or analyzed during this study are included in this published article.

\section{Consent for publication}

Not applicable.

\section{Ethics approval and consent to participate}

Prior patients' consent and ethics approval were obtained from Gynaecology Ward of Maternal and Child Health Hospital (Zaozhuang, Shandong Province, China) and Xuzhou Central Hospital.

\section{Funding}

This study was supported by Combined research of the Science and Technology department of Yunnan Province combine with Kunming Medical University (No: 2017FE467(-062) to L.Y.) and Study on the natural drug therapy for ovarian cancer (No. D-201633 to L.Y.).

\section{Publisher's Note}

Springer Nature remains neutral with regard to jurisdictional claims in published maps and institutional affiliations.

Received: 2 April 2018 Accepted: 26 February 2019

Published online: 03 April 2019

\section{References}

1. Bearfoot JL, Choong DY, Gorringe KL, Campbell IG. Genetic analysis of cancer-implicated MicroRNA in ovarian cancer. Clin Cancer Res. 2008:14(22):7246-50.

2. Dahiya N, Sherman-Baust CA, Wang TL, Davidson B, Shih le M, Zhang Y, Wood W 3rd, Becker KG, Morin PJ. MicroRNA expression and identification of putative miRNA targets in ovarian cancer. PLoS ONE. 2008;3(6):e2436.

3. Guo LM, Pu Y, Han Z, Liu T, Li YX, Liu M, Li X, Tang H. MicroRNA-9 inhibits ovarian cancer cell growth through regulation of NF-kappaB1. FEBS J. 2009;276(19):5537-46.

4. Lu L, Schwartz P, Scarampi L, Rutherford T, Canuto EM, Yu H, Katsaros D. MicroRNA let-7a: a potential marker for selection of paclitaxel in ovarian cancer management. Gynecol Oncol. 2011;122(2):366-71.

5. Rafehi S, Ramos Valdes Y, Bertrand M, McGee J, Prefontaine M, Sugimoto A, DiMattia GE, Shepherd TG. TGFbeta signaling regulates epithelial-mesenchymal plasticity in ovarian cancer ascites-derived spheroids. Endocr Relat Cancer. 2016;23(3):147-59.

6. Tan DS, Agarwal R, Kaye SB. Mechanisms of transcoelomic metastasis in ovarian cancer. Lancet Oncol. 2006;7(11):925-34

7. Yeung TL, Leung CS, Yip KP, Au Yeung CL, Wong ST, Mok SC. Cellular and molecular processes in ovarian cancer metastasis. a review in the theme: cell and molecular processes in cancer metastasis. Am J Physiol Cell Physiol. 2015;309(7):444-56.

8. Weidle UH, Birzele F, Kollmorgen G, Nopora A. Potential microRNA-related targets for therapeutic intervention with ovarian cancer metastasis. Cancer Genomics Proteomics. 2018;15(1):1-15.

9. Shan C, Ma Y. MicroRNA-126/stromal cell-derived factor 1/C-X-C chemokine receptor type 7 signaling pathway promotes post-stroke angiogenesis of endothelial progenitor cell transplantation. Mol Med Rep. 2018;17(4):5300-5.

10. Feng X, Liu N, Deng S, Zhang D, Wang K, Lu M. miR-199a modulates cisplatin resistance in ovarian cancer by targeting Hif1alpha. Oncol Targets Ther. 2017;10:5899-906

11. Zhang JG, Shi Y, Hong DF, Song M, Huang D, Wang CY, Zhao G. MiR-148b suppresses cell proliferation and invasion in hepatocellular carcinoma by targeting WNT1/beta-catenin pathway. Sci Rep. 2015;5:8087.
12. Ni CW, Qiu H, Jo H. MicroRNA-663 upregulated by oscillatory shear stress plays a role in inflammatory response of endothelial cells. Am J Physiol Heart Circ Physiol. 2011;300(5):H1762-9.

13. Hu H, Li S, Cui X, Lv X, Jiao Y, Yu F, Yao H, Song E, Chen Y, Wang M, Lin L. The overexpression of hypomethylated miR-663 induces chemotherapy resistance in human breast cancer cells by targeting heparin sulfate proteoglycan 2 (HSPG2). J Biol Chem. 2013;288(16):10973-85.

14. Pan J, Hu H, Zhou Z, Sun L, Peng L, Yu L, Sun L, Liu J, Yang Z, Ran Y. Tumorsuppressive mir-663 gene induces mitotic catastrophe growth arrest in human gastric cancer cells. Oncol Rep. 2010;24(1):105-12.

15. Yi C, Wang Q, Wang L, Huang Y, Li L, Liu L, Zhou X, Xie G, Kang T, Wang H, Zeng M, Ma J, Zeng Y, Yun JP. MiR-663, a microRNA targeting p21(WAF1/ CIP1), promotes the proliferation and tumorigenesis of nasopharyngeal carcinoma. Oncogene. 2012;31(41):4421-33.

16. Cao X, Zhao Y, Wang J, Dai B, Gentile E, Lin J, Pu X, Ji L, Wu S, Meraz I, Majidi M, Roth JA. TUSC2 downregulates PD-L1 expression in non-small cell lung cancer (NSCLC). Oncotarget. 2017;8(64):107621-9.

17. Dai B, Yan S, Lara-Guerra H, Kawashima H, Sakai R, Jayachandran G, Majidi M, Mehran R, Wang J, Bekele BN, Baladandayuthapani V, Yoo SY, Wang Y, Ying J, Meng F, Ji L, Roth JA. Exogenous restoration of TUSC2 expression induces responsiveness to erlotinib in wildtype epidermal growth factor receptor (EGFR) Lung cancer cells through context specific pathways resulting in enhanced therapeutic efficacy. PLoS ONE. 2015;10(6):e0123967

18. Xin J, Zhang XK, Xin DY, Li XF, Sun DK, Ma YY, Tian LQ. FUS1 acts as a tumor-suppressor gene by upregulating miR-197 in human glioblastoma. Oncol Rep. 2015;34(2):868-76.

19. Akcakaya P, Ekelund S, Kolosenko I, Caramuta S, Ozata DM, Xie H, Lindforss $U$, Olivecrona $H$, Lui WO. miR-185 and miR-133b deregulation is associated with overall survival and metastasis in colorectal cancer. Int J Oncol. 2011:39(2):311-8.

20. Acharyya S, Oskarsson T, Vanharanta S, Malladi S, Kim J, Morris PG, Manova-Todorova K, Leversha M, Hogg N, Seshan VE, Norton L, Brogi E, Massague J. A CXCL1 paracrine network links cancer chemoresistance and metastasis. Cell. 2012;150(1):165-78.

21. Rutnam ZJ, Du WW, Yang W, Yang X, Yang BB. The pseudogene TUSC2P promotes TUSC2 function by binding multiple microRNAs. Nat Commun. 2014;5:2914.

22. Kondo M, Ji L, Kamibayashi C, Tomizawa Y, Randle D, Sekido Y, Yokota J, Kashuba V, Zabarovsky E, Kuzmin I, Lerman M, Roth J, Minna JD. Overexpression of candidate tumor suppressor gene FUS1 isolated from the 3 p21.3 homozygous deletion region leads to G1 arrest and growth inhibition of lung cancer cells. Oncogene. 2001;20(43):6258-62.

23. Rimkus T, Sirkisoon S, Harrison A, Lo HW. Tumor suppressor candidate 2 (TUSC2, FUS-1) and human cancers. Discov Med. 2017;23(128):325-30.

24. Bi L, Yang Q, Yuan J, Miao Q, Duan L, Li F, Wang S. MicroRNA-127-3p acts as a tumor suppressor in epithelial ovarian cancer by regulating the BAG5 gene. Oncol Rep. 2016;36(5):2563-70.

25. Chen H, Zhang L, Zhang L, Du J, Wang H, Wang B. MicroRNA-183 correlates cancer prognosis, regulates cancer proliferation and bufalin sensitivity in epithelial ovarian caner. Am J Transl Res. 2016;8(4):1748-55.

26. Feng ZY, Xu XH, Cen DZ, Luo CY, Wu SB. miR-590-3p promotes colon cancer cell proliferation via Wnt/beta-catenin signaling pathway by inhibiting WIF1 and DKK1. Eur Rev Med Pharmacol Sci. 2017;21(21):4844-52.

27. Liang S, Zhang N, Deng Y, Chen L, Zhang Y, Zheng Z, Luo W, Lv Z, Li S, Xu T. miR-663b promotes tumor cell proliferation, migration and invasion in nasopharyngeal carcinoma through targeting TUSC2. Exp Ther Med. 2017;14(2):1095-103

28. Zhou YB, Huang ZX, Ren CP, Zhu B, Yao KT. Screening and preliminary analysis of the apoptosis- and proliferation-related genes in nasopharyngeal carcinoma. Nan Fang Yi Ke Da Xue Xue Bao. 2009;29(4):645-7. 